\title{
Electrical Stimulation Optimization in Bioreactors for Tissue Engineering Applications
}

\section{Paula Pascoal-Faria ${ }^{1,2, a^{*}}$, Pedro Castelo Ferreira ${ }^{1, b}$, Abhishek Datta $^{3,4, c}$, Sandra Amado ${ }^{1}$, Carla Moura ${ }^{1}$ and Nuno Alves ${ }^{1,5, d}$}

\author{
${ }^{1}$ Centre for Rapid and Sustainable Product Development, Polytechnic Institute of Leiria, Rua de \\ Portugal, Marinha Grande, 2430-028, Portugal
}

${ }^{2}$ Department of Mathematics, School of Technology and Management, Polytechnic Institute of Leiria, Rua General Norton de Matos, Apartado 4133, 2411-901 Leiria, Portugal

\author{
${ }^{3}$ Soterix Medical, Inc. \\ ${ }^{4}$ City College of New York, NY
}

${ }^{5}$ Department of Mechanical Engineering, School of Technology and Management, Polytechnic Institute of Leiria, Rua General Norton de Matos, Apartado 4133, 2411-901 Leiria, Portugal

apaula.faria@ipleiria.pt (corresponding author)

Keywords: tissue engineering, bioreactor, scaffold, electrical stimulation, electro-mechanical strain stimulation, optimization.

\begin{abstract}
We review here the current research status on bioreactors for tissue engineering with cell electrical stimulation. Depending on the cell types, electrical stimulation has distinct objectives: 1) being employed both to mimic and enhance endogenous electricity measured in the natural regeneration of living organisms and 2) to mimic strain working conditions for contractible tissues (for instance muscle and cardiac tissues). Understanding the distinct parameters involved in electrical stimulation is crucial to optimize its application. The results presented in the literature and reviewed here reveal that the application of electrical stimulation can be essential for tissue engineering applications.
\end{abstract}

\section{Introduction}

Tissue Engineering is today seen as the ultimate solution in the treatment and replacement of lost or damaged tissues. Understanding the mechanisms underlying biological tissue differentiation, regeneration and growth is a timely goal. In particular, the proper identification of the relevant parameters involved and the respective possible optimization strategies for each tissue type is a common objective in this field of research. It has been established that the following conditions should generally be satisfied:

i) an adequate mechanical laminar shear stress of the culture medium fluid that not only ensures nutrient and oxygen delivery but removal of cell activity byproducts as well $[1,2]$;

ii) an adequate low voltage and low intensity electrical stimulus that both mimics endogenous real organism conditions [3-5] and selectively enhances or inhibits cell regeneration. This type of stimulus evidently plays an essential role in artificial control of cellular differentiation [6], replication [7], growth [7, 8], maturation [9], adhesion and orientation [10].

iii) for the particular case of muscular and cardiac tissues, electro-mechanical strain stimulation mimicking the biological strain working conditions (contraction and elongation). It should be noted that the electric impulses considered in this study should not be confused with the endogenous regenerated electric fields referred in point (i), instead they refer to the functional activation of muscle and cardiac tissues of biological organisms. The scaffolds for these tissue types are commonly denominated constructs (when requiring a cell culture for construction of the scaffold) being itself constituted by biological tissues. Typically the constructs mimic the elasticity of the final biological 
tissue [11-14]. Mechanical strain alone has also been considered for cartilaginous, blood vessel and ligament tissues;

iv) the existence of a geometric adequate substrate that mimics the final desired shape and material properties. This is commonly solved by cultivating the tissue on a biodegradable scaffold which both in vitro and in vivo shapes the tissue to the intended biological shape [15-17]. Also the geometry of these scaffolds allow for medium perfusion ensuring adequate volumetric nutrient and oxygen delivery [18-20].

Regarding materials, numerous research have proposed using both natural and synthetic materials for the design and construction of tissues, including naturally occurring polymers, synthetic bioresorbable polymers or ceramics and biodegradable materials. Examples using polycaprolactone (PCL) matrix as reference, PCL reinforced with cellulose nanofibers (CNF) and PCL reinforced with $\mathrm{CNF}$ and hydroxyapatite nanoparticles have been developed and used by Morouço and collaborators (see [16]). Moreover, advances have been made in exploring the ideal geometries of scaffolds [21], mostly due to $3 \mathrm{D}$ printing methods that have been developed over the years such as fused deposition modelling, selective laser melting or selective laser sintering techniques [22, 23]. More recently, a 4D bioprinting technology that fabricates dynamic structures that improves cartilage and bone regeneration has also been proposed $[22,24]$.

Different types of stimulation have been used to benefit tissue regeneration applications over the years. Mechanical, electrical and magnetic stimulation have all been explored with promising results. However, numerical studies aiming to optimize the different stimulation parameters are critically needed focusing on the vast applications of tissue engineering. Early modelling studies performed by [25] for mechanical stimulation, by [26-28] and by [29] for electrical and magnetic stimulation have proven to be not only crucial for understanding the neurophysiological mechanics involved but have also helped to optimize the application of stimulation in different type of cells and models.

With the objective of optimizing in vitro tissue engineering for eventual in vivo chirurgical implantation in such a way that it is both economically viable and scalable to industrial levels, it is highly desirable to develop bioreactors that incorporate all the necessary conditions for tissue engineering. This review will therefore mainly focus on the research status of such bioreactors which already contemplate aforementioned culture conditions (i), (ii), (iii) and (iv); specifically we will address studies that deal with the application of electrical stimulation on a scaffold placed inside a bioreactor. Furthermore, this review aims to provide insight on the different parameters involved and suggest what future directions to take.

\section{Methods}

Information sources and literature search. Studies in English quantitatively assessing the application of electrical stimulation on a scaffold placed inside a bioreactor for tissue regeneration were identified by searching the Science Direct and PubMed electronic databases on November 12, 2017. The following search string was used to retrieve relevant publications: "("bioreactor" OR "dynamic cell culture") AND ("scaffold" OR "construct") AND ("regenerative medicine" OR "tissue engineering" OR "tissue regeneration") AND (stimuli OR stimulation) AND ("electric" OR "electrical")".

Selection and exclusion criteria. The publications retrieved by using the aforementioned search string were assessed for eligibility. Notably, for an article to be included in the qualitative analysis performed in this review, the following criteria had to be met: (1) published in English, (2) original research reports - e.g., reviews were excluded and (3) used electrical stimulation applied on cells placed on scaffolds inside bioreactors corresponding to cell culture conditions (ii) or (iii).

Additionally, the following exclusion criteria were considered: (1) case studies, (2) different types of stimulation that do not include electrical stimulation and (3) when cell culture was not directly studied.

Data extraction. Relevant data were extracted systematically from the selected articles and recorded on an extraction sheet (see Table 1). This procedure was pilot-tested independently by all the authors 
to ensure that data extraction occurred consistently. The extraction sheet included several topics, notably information regarding cell preparation, characteristics of the scaffolds and bioreactors and the different parameters involved in stimulation.

Identification of articles. After all data were collected, all the authors were involved in the preliminary screening of the papers, which consisted of assessing their specific titles and abstracts for eligibility. Each article was assessed by two authors. Whenever disagreement occurred, the paper was not excluded without both authors reaching an agreement on the reason for exclusion. The full-text version of the remaining articles was then assessed. This second stage eligibility screening was performed by the same authors and used the same method except for the disagreements, which were solved by joint discussions of all the authors.

\section{Results}

Study Selection. The search strategy described above resulted in 66 results from Science Direct and 14 from PubMed. After discarding duplicate records, reviews and book chapters, 21 articles were assessed for eligibility on the basis of their titles, abstracts and keywords, with 9 being excluded from the review (see Figure 1). Out of the remaining 12, which had their full text analyzed; an additional 2 papers were also excluded-due to non-inclusion of either culture conditions (ii) and (iii). The process was repeated by analyzing the full-text of the remaining 10 articles [14, 30-36, [37,38]. The characteristics of these articles most relevant to this review were collected on an extraction sheet as described above (see Table 1).

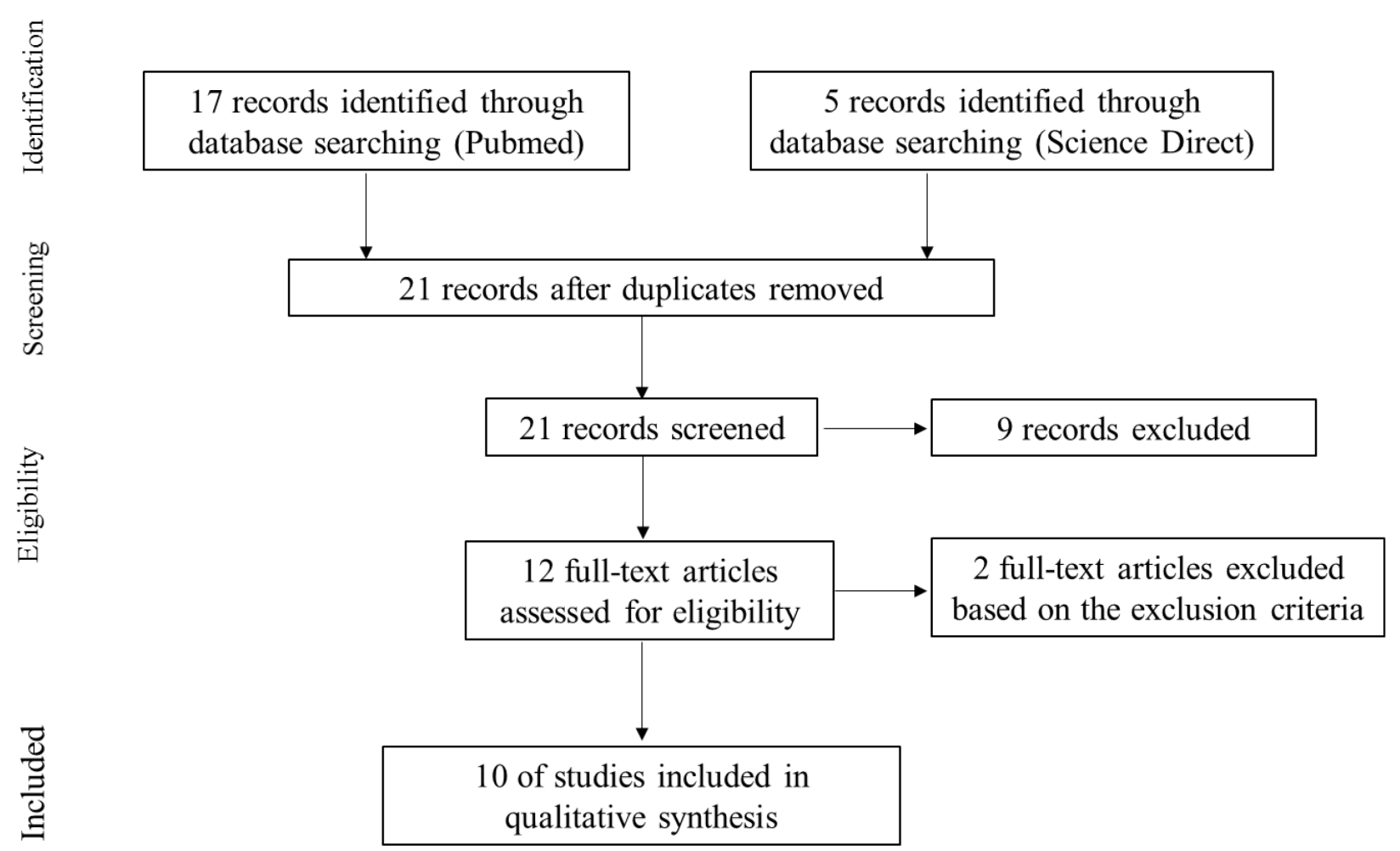

Figure 1. Flow chart diagram of the study selection process.

Characteristics of the studies. From the selected studies, [30, 37] correspond to tissue cultures applying low electrical current stimulation (ii) while the studies [14, 31-36, 38] correspond to electromechanical strain stimulation (iii). 


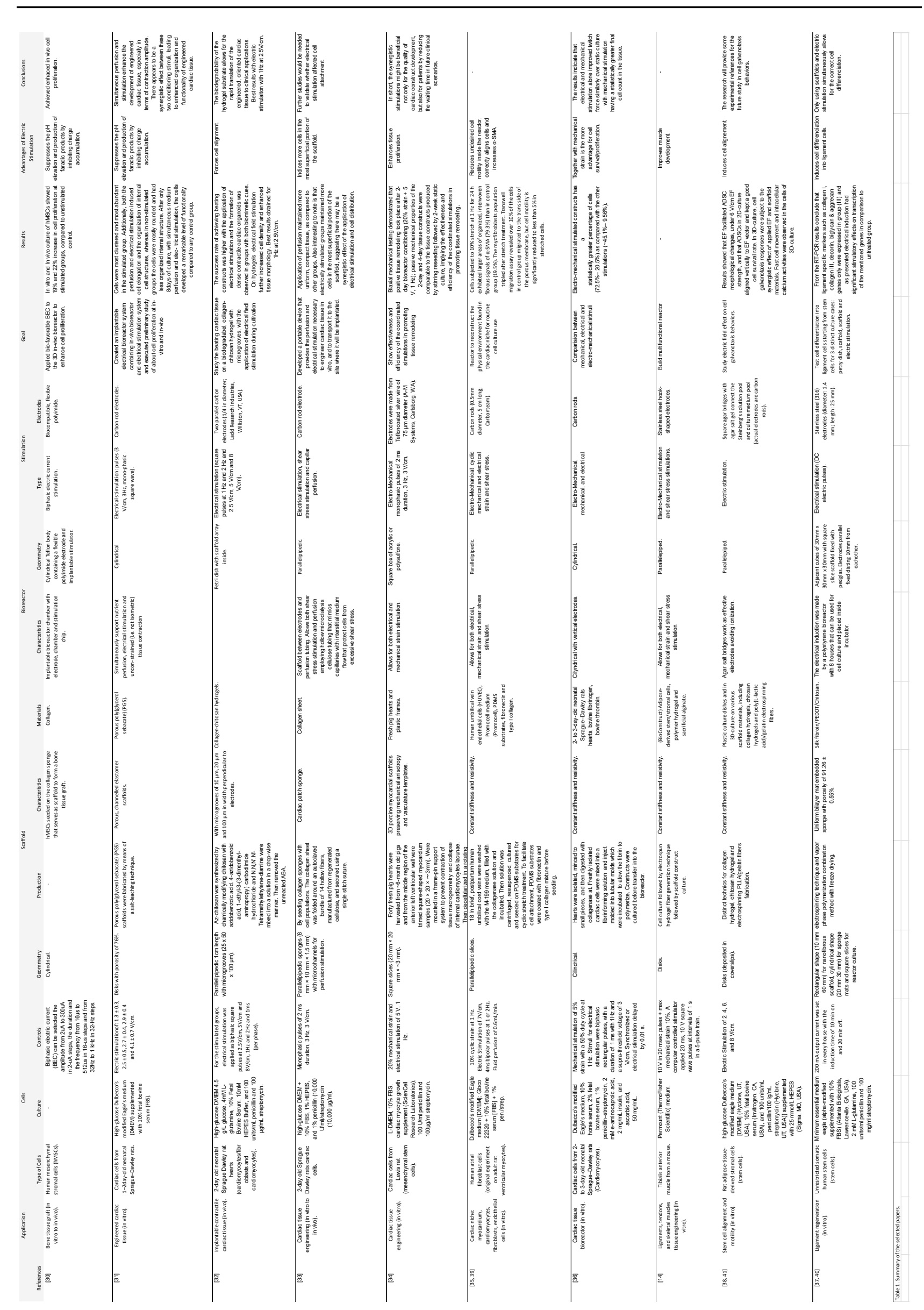


Types of cell tissues. As for the cell types considered in each of the selected references, six studies deal with cardiac tissue engineering [31-36, 38], one with bone tissue engineering [30], one with muscle/ligament/tendon tissue engineering [14] and two with stem cell tissue engineering, [38]. Regarding the objective of generic cell differentiation, Dodel and his collaborators (see [37]) explicitly show that differentiation into ligament tissue requires electrical stimulation. In all these studies, it has been shown experimentally that the electrical stimulation and electro-mechanic stimulation is beneficial to the cell culture with respect to the control cultures without any electrical or mechanical stimulation. The interpretation is therefore common among all these studies, the stimuli mimic the natural organism conditions of tissues. In particular the cardiac tissue is naturally strain stimulated in living organisms as well as muscle, ligament and tendon tissues. We highlight the study [36] where electric strain, mechanical strain and both electro-mechanical stimulations are compared for cardiac tissue with the conclusion that electro-mechanical strain with electric stimulation when delayed with respect to the mechanical stimulation is the more beneficial stimuli for cell health. Not surprisingly this is indeed the stimulation that more closely resembles the working conditions of cardiac tissue in living organisms.

Scaffold Characteristics. The scaffolds employed in the reactors are commonly made out of a mixture of both non-biological and biological cell tissues (synthesized and/or natural). The biological components range from generic components as collagen [30,33], chitosan [37, 38], collagen-chitosan hydrogels [32, 38], poly(glycerol sebacate) [31] and poly(l-lactic acid) [38] to specific tissues adequate as substrate for each target cell culture. These tissues range from fresh pig hearts [34] (cardiac), human umbilical vein endothelial cells [35] (cardiac), rat hearts [33, 36] (cardiac) to adipose-derived stem/stromal cells [14] (ligament differentiation). The non-biological components for the scaffolds are commonly polymers as discussed in the introduction.

Synthetic scaffolds were employed in [30-32, 37]. When required to have a cell culture for the construction of the scaffolds, these are typically denominated as constructs $[33-36,14,38]$. The fabrication process employed includes cell culture after mixture/deposition in the base substrate [33$36,38]$ and electrospinning [14, 37].

The specific shape of the scaffolds depend on the application: [31-35] considered parallelipipedic / square slices, [36, 37] considered cylindrical, and finally [14, 38, 37] considered disk shaped scaffolds.

Bioreactor. Each bioreactor has distinct objectives while in [31, 34-36, 14, 38] cell culture was studied strictly in vitro, in [32] cell culture was studied strictly in vivo, in $[30,33]$ the goal was to study the viability and optimization of the transfer of in vitro cell culture to living organisms. In particular [30] suggest a bioreactor that enhances cell proliferation in vivo and in [33] it is shown that mechanical perfusion and electrical stimulation in vitro prior to transference of cell cultures to living organisms improves cell survival rate in vivo.

The geometry of the reactors considered in the selected studies is typically either cylindrical [30$32,36]$ or parallelipipedic [33-35, 14, 38, 37] (see [39] for detailed description of [35]). When considering mechanical strain stimulation, servo actuators are typically employed [34-36, 14]. As for the electrical electrodes, composition involves either -gold [30] (wire of diameter 300um forming a pattern of $12 \mathrm{~mm}$ by $3 \mathrm{~mm}$ ), carbon [31-33, 35, 36] (rods with diameter ranging from $0.5 \mathrm{~mm}$ to $5 \mathrm{~mm}$ ), silver [34] (wire of $75 \mu \mathrm{m}$ diameter), stainless steel [38, 37] (rods of diameter $1.4 \mathrm{~mm}$, described in [40]) and salt bridges [38] (square agar bridges, the actual electrode is a carbon rod, described in [41]). It is relevant to note that the direct insertion of electrodes in the culture medium may induce ionization depending on the specific value of the voltage and current intensity. As this is undesirable for the cell culture, one possible solution is to consider salt bridges between the actual culture medium and independent recipients where the actual electrodes are immersed (see for instance [5] and references therein), being [38] the only selected bioreactor study that actually employed salt bridges. Typically for electro-mechanical strain stimulation the electrodes are in direct contact with the culture 
medium (through the reactor wells when present) [30-33, 35-37] or in direct contact with the cells/scaffold $[34,14]$.

Stimulation. Studies included in this review show that different stimulation parameters seem to play a key role on cell differentiation, regeneration and growth. Scaffold stiffness (Young's modulus) and electric resistivity: Laminar shear stress and perfusion mimicking the volumetric biological vascular systems. Also important is understanding the adhesion of cell cultures and its biodegradability. The geometry and topology of the scaffold is also crucial in the optimization of stimuli application [21, 23]. We highlight the two different types of stimulation that are mostly used and the main parameters involved:

i) Mechanical stimulation: amplitude, frequency and pulse shape, periodicity on/off during culture time and synchronization with respect to electrical stimulation were the main parameters involved;

ii) Electrical stimulation: electrodes characteristics like shape, dimensions and spacing between electrodes varied according to the different applications studied [30-33, 35-38]. Additionally, electric voltage and intensity, frequency and pulse shape, periodicity on/off during culture time and synchronization with respect to mechanical stimulation (when present) are parameters that have been shown to influence the results of electrical stimulation $[32,38]$.

\section{Discussion}

In this review paper we report in detail the influence of applying electrical stimulation on cells placed on a scaffold inside a bioreactor. Most of the papers in the literature to-date have used only mechanical stimulation. However, recently, some studies have shown the advantage of using different types of stimulation, such as, electrical or magnetic either in isolation or in combination [29, 42-44]. In order to understand the neurophysiology of the underlying mechanics, an in-depth study of all stimulation parameters involved should be performed. Initially our plan was to consider both numerical and experimental studies. However after searching on Science Direct and PubMed for relevant papers that address an updated string (the original string with addition of the keywords: simulation, numerical and modelling), we did not find any paper addressing the use of computational simulation to optimize the stimulation parameters. Therefore we decided to focus only on the experimental results. Here, we are mainly interested in understanding which type of parameters have been used for the different tissue engineering applications and possible optimizations on designs, protocols and stimulation parameters that may increase cell differentiation, growth, proliferation and survival rate. It was also possible to conclude that different electrode characteristics were considered empirically for the different applications, for instance, either they were emerged in the medium orthogonally to the scaffold or horizontally aligned with the scaffold. Additionally, electric voltage and intensity, frequency and pulse shape, periodicity on/off during culture time and synchronization with respect to mechanical stimulation (when present) are parameters that have been shown to influence the results of electrical stimulation $[32,38]$.

Once the relevance and optimization of the physical parameters for each stimulus is experimentally measured, numerical simulations can significantly reduce experimental time and costs [44]. In particular the optimal topologies and configurations of bioreactors can be investigated by numerical simulations prior to new experimental setups. For instance, Pereira et al., show numerically the importance of choosing the adequate mechanical stimuli parameters to improve shear stress value and fluid flow on the scaffold. Further, other numerical studies have investigated the influence of mechanical stimulation on the growth and distribution of cells on scaffolds and tissue differentiation [45-48].

Additionally, the developed bioscaffolds are usually tested and studied in vivo, presenting a significant expensive option thereby necessitating the development of numerical approaches. Therefore computational studies are of paramount importance to optimize the direct digital 
manufacturing of scaffolds that can be produced using different geometries, geometry gradients, topologies and also different materials.

Finally, we note that the low number of bioreactor studies including (i), (iii) and (ii) or (iv) reflects both the complexity and multidisciplinary nature of the research field as distinct conditions are required for each organic tissue and the multitude of scaffold materials and respective construction process. It is relevant to note that no studies including both regenerative electrical stimulation and electro-mechanic strain stimulation have been found, it is however expected that further enhancement for contractible tissues may be expected by considering both these stimulations.

\section{Conclusions}

Only few studies were found that focus on the application of electrical stimulation on cells placed on a scaffold inside a bioreactor. The results are promising when using an electrical stimuli and also when combining it with mechanical stimulation. An increase in cell proliferation and density was observed as well as an enhancement in tissue morphology. Additionally, further studies must be performed where stimuli responsive scaffolds are used enabling a better cell proliferation, differentiation or tissue growth. This can be achieved through the combination of different types of stimuli to optimize both the response of the intelligent scaffold and cells behavior. Numerical studies can play here a key role to optimize all the stimulation parameters involved and predict cell response which can lead to a reduction of experimental time and costs.

\section{Acknowledgments}

This work was supported by the Portuguese Foundation for Science and Technology (FCT) through the Projects references UID/Multi/04044/2013, BIOMATE (PTDC/EMS-SIS/7032/2014) and Stimuli2BioScaffold (02/SAICT/2017, POCI-01-0145-FEDER-032554). It was also funded by PAMI (ROTEIRO/0328/2013; N 022158), a Research Infrastructure of the National Roadmap of Research Infrastructures of Strategic Relevance for 2014-2020, co-funded by the Portuguese Foundation for Science and Technology (FCT) and European Union through the Centro2020 and MATIS (CENTRO-01-0145-FEDER-000014 - 3362) funded by the European Union through the PT2020 and Centro2020.

\section{References}

[1] G. Vunjak-Novakovic, I. Martin, B. Obradovic, S. Treppo, A. J. Grodzinsky, R. Langer and L. E. Freed, Bioreactor Cultivation Conditions Modulate the Composition and Mechanical Properties of Tissue-Engineered Cartilage, J Orthop. Res. 17 (1999) 130.

[2] R. L. Carrier, M. Papadaki, M. Rupnick, F. J. Schoen, N. Bursac, R. Langer, L. E. Freed, G. Vunjak-Novakovic, Cardiac tissue engineering: Cell seeding, cultivation parameters, and tissue construct characterization, Biotechnol. Bioeng. 64 (1999) 580.

[3] DuBois-Reymond, E. Vorla ufiger Abriss einer Untersuchung uber den sogenannten Froschstrom und die electomotorischen Fische. Ann. Phy. U. Chem. 58 (1843) 1-30.

[4] R. B. Borgen, What Is the Role of Naturally Produced Electric Current in Vertebrate Regeneration and Healing?, International Review of Cytology, 76 (1982) 245.

[5] B. Song, Y. Gu, J. Pu, B. Reid, Z. Zhao and M. Zhao, Application of direct current electric fields to cells and tissues in vitro and modulation of wound electric field in vivo, Nature Protocols 2 (2007) 1479.

[6] F. Pires, Q. Ferreira, C. A.V. Rodrigues, J. Morgado, F. Castelo Ferreira, Neural stem cell differentiation by electrical stimulation using a cross-linked PEDOT substrate: Expanding the use of biocompatible conjugated conductive polymers for neural tissue engineering, Biochimica et Biophysica Acta 1850 (2015) 1158-1168. 
[7] N. Selvamurugan, Z. He, D. Rifkin, B. Dabovic and N. C. Partridge, Pulsed Electromagnetic Field Regulates MicroRNA 21 Expression to Activate TGF- $\beta$ Signaling in Human Bone Marrow Stromal Cells to Enhance Osteoblast Differentiation, Stem Cells International 2017 (2017) 2450327.

[8] A. N. Koppes, A. M. Seggio and D. M. Thompson, Neurite outgrowth is significantly increased by the simultaneous presentation of Schwann cells and moderate exogenous electric fields, J. Neural Eng. 8 (2011) 046023.

[9] G. H. Jin and G. H. Kim, The effect of sinusoidal AC electric stimulation of $3 D P C L / C N T$ and PCL/b-TCP based bio-composites on cellular activities for bone tissue regeneration, J. Mater. Chem. B1 (2013) 1439.

[10] R. Balint, N. J. Cassidy and S. H. Cartmell, Electrical Stimulation: A Novel Tool for Tissue Engineering, Tissue Engineering: Part B19 (2013) 48.

[11] N. Bursac, M. Papadaki, R. J. Cohen, F.J. Schoen, S. R. Eisenberg, R. Carrier, G. VunjakNovakovic, L. E. Freed, Cardiac muscle tissue engineering: toward an in vitro model for electrophysiological studies, Am J Physiol. 277 (1999) H433.

[12] W.-H. Zimmermann, C. Fink, D. Kralisch, U. Remmers, J. Weil, T. Eschenhagen, Threedimensional engineered heart tissue from neonatal rat cardiac myocytes, Biotechnol. Bioeng. 68 (2000) 106-14.

[13] G. H. Altman, R. L. Horan, P. R. H. Stark, I. Martin, J. Farhadi, J. C. Richmond, G. VunjakNovokovic and D. L. Kaplan, Cell Differentiation by Mechanical Stress, FASEB J. 16 (2001) 270272; G H. Altman et al., Advanced Bioreactor with Controlled Application of Multi-Dimensional Strain For Tissue Engineering, Transactions of the ASME 124 (2002) 742.

[14] C. A. Cook, P. Y. Huri, B. P. Ginn, J. Gilbert-Honick, S. M. Somers, J. P. Temple, H.-Q. Mao and W. L. Grayson, Characterization of a Novel Bioreactor System for 3D Cellular Mechanobiology Studies, Biotechnology and Bioengineering 113 (2016) 1825-1837.

[15] G. H. Jin, G.-H. Yang, G. H. Kim, Tissue engineering bioreactor systems for applying physical and electrical stimulations to cells, Journal of Biomedical Materials Research Part B103 (2015) 935948.

[16] P. Morouço, S. Biscaia, T. Viana, M. Franco, C. Malça, A. Mateus, C. Moura, F. Castelo Ferreira, G. Mitchell and N. Alves, Fabrication of Poly(-caprolactone) Scaffolds Reinforced with Cellulose Nanofibers, with and without the Addition of Hydroxyapatite Nanoparticles, BioMed Research International 2016 (2016) 1596157.

[17] P. Morouço, W. Lattanzi and N. Alves, Four-Dimensional bioprinting as a new era for tissue engineering and regenerative medicine, Front. Bioeng. Biotechnol. 17 (2017) 61.

[18] S S Kim, H Utsunomiya, J A Koski, B M Wu, M J Cima, J Sohn, K Mukai, L G Griffith, and J $\mathrm{P}$ Vacanti, Survival and function of hepatocytes on a novel three-dimensional synthetic biodegradable polymer scaffold with an intrinsic network of channels, Ann Surg. 228 (1998) 8-13.

[19] M. Radisic, M. Euloth, L. Yang,R. Langer, L. E. Freed, G. Vunjak-Novakovic, High-Density Seeding of Myocyte Cells for Cardiac Tissue Engineering, Biotechnol. Bioeng. 82 (2003) 403.

[20] A. Marsano, R. Maidhof, N. Tandon, J. Gao, Y. Wang, G. Vunjak-Novakovic, Engineering of functional contractile cardiac tissues cultured in a perfusion system, 30th Annual International IEEE EMBS Conference 2008 (2008) 3590.

[21] A. A. Zadpoor, Bone tissue regeneration: the role of scaffold geometry, Biomater. Sci. 3 (2015) 231-245.

[22] Y.-C. Li, Y. S. Zhang, A. Akpek, S. R. Shin and A. Khademhosseini, 4D bioprinting: the nextgeneration technology for biofabrication enabled by stimuli-responsive materials, Biofabrication 9 (2017) 012001. 
[23] R. L. Truby and J. A. Lewis, Printing soft matter in three dimensions, Nature 540 (2016) 371378.

[24] B. Gao, Q. Yang, X. Zhao, G. Jin, Y. Ma, and F. Xu, 4D Bioprinting for Biomedical Applications, Trends in Biotechonology 34 (2016) 746-756.

[25] R. Pereira, D. Freitas, A. Tojeira, H. Almeida, N. Alves, P. Bártolo, Computer modelling and simulation of a bioreactor for tissue engineering, International Journal of Computer Integrated Manufacturing, 27 (2013) 1-14.

[26] P. Faria, P. Cavaleiro Miranda, Alberto Leal, Comparing different electrode configurations using the 10-10 international system in tDCS: a finite element model analysis, Conf Proc. IEEE Eng. Med. Biol. Soc. 2009 (2009) 1:1596-9.

[27] P. Cavaleiro Miranda, P. Faria and M. Hallet, What does the ratio of injected current to electrode area tell us about current density in the brain during tDCS, Clinical Neurophysiology, 120 (2009) 1183-87.

[28] P. Faria, M. Hallet, P. Cavaleiro Miranda, A finite element analysis of the effect of electrode area and inter-electrode distance on the spatial distribution of the current density in tDCS, Journal of Neural Engineering. 8 (2011) 066017.

[29] R. Salvador, C. Wenger and P. C. Miranda, Investigating the cortical regions involved in MEP modulation in tDCS, Front Cell Neurosci, 9 (2015) 405.

[30] J. Hoon Kim, T. Hyung Lee, Y. Mi Song, I. Sook Kim, T. Hyung Cho, S. Jung Hwang and S. June Kim., An Implantable Electrical Bioreactor for Enhancement of Cell Viability, 33rd Annual International Conference of the IEEE EMBS (2011) 3601.

[31] R. Maidhof, N. Tandon, E. Jung Lee, J. Luo1, Y. Duan, K. Yeager, E. Konofagou and G. VunjakNovakovic, Biomimetic perfusion and electrical stimulation applied in concert improved the assembly of engineered cardiac tissue, J Tissue Eng Regen Med 6 (2011) e12-e23.

[32] L.L. Y. Chiu, K. Janic and M. Radisic, Engineering of oriented myocardium on threedimensional micropatterned collagen-chitosan hydrogel, Int. J. Organs 35 (2012) 237-250.

[33] N. Tandon, A. Taubman, E. Cimetta, L. Saccenti, and G. Vunjak-Novakovic, Portable bioreactor for perfusion and electrical stimulation of engineered cardiac tissue, Conf. Proc. IEEE Eng Med Biol Soc. 2013 (2013) 6219-6223.

[34] B. Wang, G. Wang, F. To, J. R. Butler, A. Claude, R. M. McLaughlin, L. N. Williams, A. L. de Jongh Curry and J. Liao, Myocardial Scaffold-based Cardiac Tissue Engineering: Application of Coordinated Mechanical and Electrical Stimulations, Langmuir 29 (2013) 11109-11117.

[35] L. Lu and U. Ravens, The use of a novel cardiac bioreactor system in investigating fibroblast physiology and its perspectives, Organogenesis 9 (2013) 82-86.

[36] K. Ye Morgan and L. Deems Black, Mimicking Isovolumic Contraction with Combined Electromechanical Stimulation Improves the Development of Engineered Cardiac Constructs, Tissue Engineering: Part A 20 (2014) 1654-1667.

[37] M. Dodel, N. Hemmati Nejad, S. Hajir Bahrami, M. Soleimani, L. Mohammadi Amirabad, H. Hanaee-Ahvaz, A. Atashi, Electrical stimulation of somatic human stem cells mediated by composite containing conductive nanofibers for ligament regeneration, Biologicals 46 (2017) 99-107.

[38] G. Yang, H. Long, X. Ren, K. Ma, Z. Xiao, Y. Wang and Y. Guo, Regulation of adipose-tissuederived stromal cell orientation and motility in $2 D$-and $3 D$-cultures by direct-current electrical field, Develop. Growth Differ. 59 (2017), 70-82. 
[39] L. Lu, M. Mende, X. Yang, H.-F. Körber, H.-J. Schnittler, S. Weinert, J. Heubach, C. Werner and U. Ravens, Design and Validation of a Bioreactor for Simulating the Cardiac Niche: A System Incorporating Cyclic Stretch, Electrical Stimulation, and Constant Perfusion, Tissue Engineering: Part A19 (2013) 403-414.

[40] L. M. Amirabad, M. Massumi, M. Shamsara, I. Shabani, A. Amari, M. M. Mohammadi, S. Hosseinzadeh, S. Vakilian, S. K. Steinbach, M. R. Khorramizadeh, M. Soleimani, and J. Barzin, Enhanced cardiac differentiation of human CVD patient specific iPS cells by applying unidirectional electrical pulses using aligned electroactive nanofibrous scaffolds, ACS Appl. Mater. Interfaces, 9 (2017) 6849-6864.

[41] H. Long, G. Yang and Z. Wang, Galvanotactic Migration of EA.Hy926 Endothelial Cells in a Novel Designed Electric Field Bioreactor, Cell Biochem Biophys 61 (2011)481-491.

[42] S.M. Ross, Combined DC and ELF Magnetic Fields Can Alter Cell Proliferation, Bioelectrornagnetics 11 (1990) 27-36.

[43] G. S. Pell, Y. Roth and A. Zangen, Modulation of cortical excitability induced by repetitive transcranial magnetic stimulation: Influence of timing and geometrical parameters and underlying mechanisms, Progress in Neurobiology 93 (2011) 59-98.

[44] P. C. Grunert, A. Jonitz-Heincke, Y. Su, R. Souffrant, D. Hansmann, H. Ewald, A. Krüger, W. Mittelmeier, R. Bader, Establishment of a Novel In Vitro Test Setup for Electric and Magnetic Stimulation of Human Osteoblasts, Cell Biochem. Biophys. 70 (2014) 805-817.

[45] M. Cioffi, J. Ku ffer, S. Stro bel, G. Dubini, I. Martin and D. Wendt, Computational evaluation of oxygen and shear stress distributions in 3D perfusion culture systems: Macro-scale and microstructured models, Journal of Biomechanics 41 (2008) 2918-2925.

[46] D. W. Hutmacher and H. Singh, Computational fluid dynamics for improved bioreactor design and $3 D$ culture, Trends in Biotechnology 26 (2008) 166.

[47] A. Sohail, K. Maqbool, A. Asif, H. Ahmad, Numerical Modelling of Effective Diffusivity in Bone Tissue Engineering, World Academy of Science, Engineering and Technology International Journal of Agricultural and Biosystems Engineering 9 (2015) 84.

[48] E. G. F. Mercuri, A. L. Daniel, M. B. Hecke and L. Carvalho, Influence of different mechanical stimuli in a multi-scale mechanobiological isotropic model for bone remodelling, Med. Eng. Phys. 38 (2016) 904-910. 